\title{
Sijunzi decoction-treated rat serum induces apoptosis of side population cells in gastric carcinoma
}

\author{
JIANGUANG JIA $^{1 *}$, YIYU QIN ${ }^{2,3 *}$, LIGONG ZHANG ${ }^{1}$, \\ CHENXU GUO $^{1}$, YAGUO WANG ${ }^{1}$, XICHENG YUE ${ }^{1}$ and JUN QIAN ${ }^{1}$ \\ ${ }^{1}$ Department of Oncology Surgery, The First Affiliated Hospital of Bengbu Medical College, Bengbu, Anhui 233003; \\ ${ }^{2}$ Department of Science and Technology, Jiading Central Hospital, Shanghai 201800; ${ }^{3}$ Department of Science \\ and Technology, Yancheng Institute of Health Sciences, Yancheng, Jiangsu 224005, P.R. China
}

Received September 20, 2015; Accepted November 25, 2016

DOI: $10.3892 / e t m .2017 .5560$

\begin{abstract}
Sijunzi decoction (SJZD) is a traditional Chinese herbal medicine. Previous studies have indicated that SJZD exhibits antitumor activity. However, the underlying molecular mechanism has not been fully elucidated. To explore the antitumor mechanism of SJZD, the effects of serum from rats treated with SJZD on the proliferation of MKN-28 and HGC-27 gastric carcinoma cell lines were systematically investigated. It was found that SJZD-treated rat serum significantly inhibited the growth of MKN-28 and HGC-27 cells in vitro. The results obtained from a colony formation assay showed that SJZD-treated rat serum decreased the colony formation ability of MKN-28 and HGC-27 cells. The apoptosis rate in MKN-28 and HGC-27 cells was also increased following treatment with SJZD-treated rat serum. Flow cytometry with cell sorting revealed the presence of side population (SP) cells in MKN-28 and HGC-27 cells though Hoechst 33342 staining, and verapamil reduced the SP percentage. Further analysis showed that SJZD-treated rat serum promoted the apoptosis of SP cells in MKN-28 and HGC-27 cell lines by upregulating Bax, caspase-3 and PARP and downregulating bcl-2. These data revealed the therapeutic effect of SJZD-treated rat serum on gastric carcinoma. Following the preliminary identification of the inhibitory effect on the growth of gastric cancer cells in vitro, the growth inhibitory effect of SJZD-treated rat serum on SP cells was confirmed, and this inhibition particularly involved the induction of cell apoptosis.
\end{abstract}

Correspondence to: Dr Jun Qian, Department of Oncology Surgery, The First Affiliated Hospital of Bengbu Medical College, 287 Changhuai Road, Bengbu, Anhui 233003, P.R. China

E-mail: 78355929@qq.com

${ }^{*}$ Contributed equally

Key words: Sijunzi decoction, gastric carcinoma, side population, apoptosis

\section{Introduction}

Chinese traditional medicine (CM) has been widely used for the prevention and treatment of cancer for many years. For patients with advanced cancer, CM, which is commonly used as an adjuvant therapy with surgery, radiotherapy, chemotherapy and other treatments, may play a role in inducing the remission of symptoms, and reducing the side effects and toxicity of chemotherapy (1). Thus, to a certain extent, CM is able to prolong the survival period and improve the life quality of patients. Moreover, compared with modern medicine, CM has the advantages of low toxicity and price, and so is suitable for long-term use (2). There are already some traditional medicines that have exhibited curative effects in the treatment of tumors. Arsenic trioxide has been applied to treat acute myelocytic leukemia, and induces apoptosis in tumor cells (3). Indigo jade red is used in the treatment of chronic myelogenous leukemia and can significantly inhibit tumor growth $(4,5)$. Matrine is able to inhibit the growth of a variety of solid tumors, and has exhibited activity against pancreatic cancer, breast cancer, lung cancer and bile duct cancer cells (6-9). In recent years, increasing numbers of Chinese herbal medicines or prescriptions have gained international recognition and acceptance for their anticancer effects (10). Therefore, exploring the application of CM prescriptions in the treatment of tumors may provide a new strategy for tumor therapy.

Sijunzi decoction (SJZD) was issued firstly in 'Taiping Huimin Heji Jufang', a Chinese pharmacopoeia of the Song Dynasty, as a representative recipe for 'reinforcing asthenia qi' and nourishing the spleen. It consists of asiabell root (Codonopsis Pilosula), white atractylodes (Atractylodes macrocephala) rhizome, Poria cocos and licorice (Glycyrrhiza uralensis) root. Clinically, SJZD has been used to treat peptic or gastric ulcer and disorders of the spleen (11). A clinical study has shown that SJZD improves the postoperative survival of patients with gastric carcinoma (12). One study demonstrated that SJZD effectively inhibited the growth of tumors in nude mice bearing SGC-7901 xenografts (13). However, that study did not carry out in vitro cell function tests, and the molecular mechanism of its antitumor effect remains unclear. Due to the complexity of the components in SJZD, it is difficult to identify the function of each component. When crude preparations 
are directly used for in vitro pharmacological experiments, the impurities, electrolytes and tannic acid components, and even the $\mathrm{pH}$ may affect the cell survival environment, leading to false negative and false positive results. In medicated serum, the preparation has undergone a series of biotransformations following digestion and absorption in the gastrointestinal tract. The medicated serum may then be added to a culture system of cells or tissues in vitro. This approach closely resembles the environment in which drugs work in vivo, where the original composition of medicated serum may be transformed into active ingredients or metabolically inactivated in vivo. Thus, the therapeutic effect of medicated serum is representative of serum pharmacology, and more befitting than the use of traditional pharmacology in traditional CM research (14). There have been a number of studies using serum from animals treated with Chinese herbal medicine in in vitro cell experiments, which have shown good applicability (15-17). In the present study, serum from Sprague-Dawley (SD) rats treated with SJZD was used to serve as an authentic mimic of in vivo circumstances. The aim was to investigate the effect of serum from SJZD-treated rats on the proliferation and apoptosis of MKN-28 and HGC-27 cells. The side population (SP) cells were investigated by the application of flow cytometric cell sorting. Furthermore, the ability of SJZD-treated rat serum to promote the apoptosis of MKN-28 and HGC-27 SP cells was analyzed. Finally, the changes of apoptosis-related proteins in SP cells treated with SJZD-treated rat serum were evaluated.

\section{Materials and methods}

Preparation of SJZD-treat rat serum. Asiabell root, white atractylodes rhizome, poria and licorice root in a ratio of 15:15:10:6 were soaked in $\mathrm{dd}_{2} \mathrm{O}$ for $30 \mathrm{~min}$. Subsequently, $400 \mathrm{ml} \mathrm{dd}_{2} \mathrm{O}$ was added and the mixture was boiled for $40 \mathrm{~min}$ until $150 \mathrm{ml}$ suspension remained. The liquor was removed by filtration and $400 \mathrm{ml} \mathrm{dd}_{2} \mathrm{O}$ was added to the residue, which was then boiled for $40 \mathrm{~min}$ until $150 \mathrm{ml}$ remained. This was filtered, and the two liquors were combined and boiled until the volume was reduced to $150 \mathrm{ml}$ (when the concentration was $0.2 \mathrm{~g} / \mathrm{ml}$ ). Subsequent boiling reduced the concentration to $2 \mathrm{~g} / \mathrm{ml}$ and this extract was stored at $4^{\circ} \mathrm{C}$.

A total of 40 female SD rats (age, 2-3 months; weight, 250-300 g; SLAC Laboratory Animal Co., Ltd., Shanghai, China) were maintained in a temperature and humidity controlled room $\left(21^{\circ} \mathrm{C}, 50 \%\right.$ humidity $)$ with a 12-h light/dark cycle in specific pathogen-free conditions. Rats were given ad libitum access to food and water. Rats were divided into four groups ( $\mathrm{n}=10$ in each group) randomly as follows: Saline-treated control, low dosage SJZD, middle dosage SJZD and high dosage SJZD. The dosages used were as follows: Middle dosage, $4.67 \mathrm{~g} / \mathrm{kg}$ body weight; high dosage, twice the middle dosage; low dosage, half the middle dosage. Prior to intragastric administration, rats were fasted for $12 \mathrm{~h}$ and intragastric administration was then performed twice a day for 3 consecutive days. Following the last intragastric administration, rats were anesthetized with urethane $(1-1.5 \mathrm{~g} / \mathrm{kg}$ intraperitoneally). Blood was collected from the abdominal aorta and serum was separated immediately and stored at $-20^{\circ} \mathrm{C}$. The current study was approved by the ethics committee of The First Affiliated Hospital of Bengbu Medical College.
Cell culture. Human gastric carcinoma cell lines MKN-28 and HGC-27 were purchased from the Institute of Biochemistry and Cell Biology, Shanghai Institutes for Biological Sciences (SIBS), Chinese Academy of Sciences (Shanghai, China). Cells were maintained at $37^{\circ} \mathrm{C}$ in Dulbecco's modified Eagle's medium (Gibco; Thermo Fisher Scientific, Inc., Waltham, MA, USA) supplemented with $10 \%$ fetal bovine serum (FBS; Sigma-Aldrich; Merck Millipore, Darmstadt, Germany), $1 \mathrm{mM}$ non-essential amino acid (Sigma-Aldrich; Merck Millipore) and 1\% penicillin/streptomycin (Gibco; Thermo Fisher Scientific, Inc.) in a $5 \% \mathrm{CO}_{2}$ incubator. Subcultures were maintained at $80 \%$ confluence and passaged with 0.25\% trypsin (Gibco; Thermo Fisher Scientific, Inc.).

MTT assay. In this assay, $5 \times 10^{3}$ cells were seeded into 96-well plates and incubated with rat serum from each of the four groups (10 or 20\%) for $72 \mathrm{~h}$. Prior to detection, cells were incubated for $4 \mathrm{~h}$ with $20 \mu \mathrm{l}(5 \mathrm{~g} / \mathrm{l}) \mathrm{MTT}$, dissolved in serum-free medium. Washing with phosphate-buffered saline (PBS; $1 \mathrm{ml}$ ) was followed by the addition of DMSO $(100 \mu \mathrm{l})$, and gentle shaking for $10 \mathrm{~min}$ so that complete dissolution was achieved. Aliquots $(200 \mu \mathrm{l})$ of the resulting solutions were transferred to 96 -well plates and the absorbance was recorded at $490 \mathrm{~nm}$ using a microplate reader (SpectraMax 190; Molecular Devices, Sunnyvale, CA, USA). The cell survival rate was calculated from optical density (OD) values using the following formula: Cell survival rate $(\%)=(O D$ experimental group-OD background)/(OD control group-OD background) $\mathrm{x} 100$.

Colony formation assay. The ability of cells to form colonies (consisting of $\geq 50$ cells) was evaluated using a colony formation assay. In this assay, $150 \mathrm{MKN}-28$ or HGC-27 cells were plated in 24-well plates and treated with rat serum from each group (10 or $20 \%$ ) for 2 weeks. Colonies were counted under an Olympus BX51 microscope (Olympus Corporation, Tokyo, Japan) following crystal violet staining, and the formation rate was calculated as follows: Colony formation rate $(\%)=$ colony number/plated number x100.

Apoptosis analysis. FITC-Annexin V (green fluorescence) and the non-vital dye propidium iodide (PI; red fluorescence) staining allows the discrimination of intact cells (FITC-PI'), early apoptotic (FITC $\left.{ }^{+} \mathrm{PI}^{-}\right)$and late apoptotic or necrotic cells $\left(\mathrm{FITC}^{+} \mathrm{PI}^{+}\right)$. These fluorescent dyes were used for analysis of the apoptotic effect of SJZD-treated rat serum on gastric carcinoma and SP cells. Briefly, cells treated with rat serum from each of the four groups (10 or 20\%) for $48 \mathrm{~h}$ were trypsinized, then rinsed with pre-cooled PBS and resuspended in $400 \mu 1$ Annexin V-FITC binding buffer at a final density of $1 \times 10^{6}$ cells/ml. Annexin V-FITC (5 $\mu \mathrm{l})$ and PI (10 $\mu \mathrm{l})$ were added to the cell suspension. The cell suspension was gently vortexed and then incubated for $5 \mathrm{~min}$ at $4^{\circ} \mathrm{C}$ with protection from light. The resulting samples were analyzed using a FACSCalibur flow cytometer (BD Biosciences, San Jose, CA, USA) within $1 \mathrm{~h}$.

Fluorescence-activated cell sorting. SPs have been identified in cancer and may efflux chemotherapy drugs, accounting for the resistance of cancer to chemotherapy (18). In the present 
A

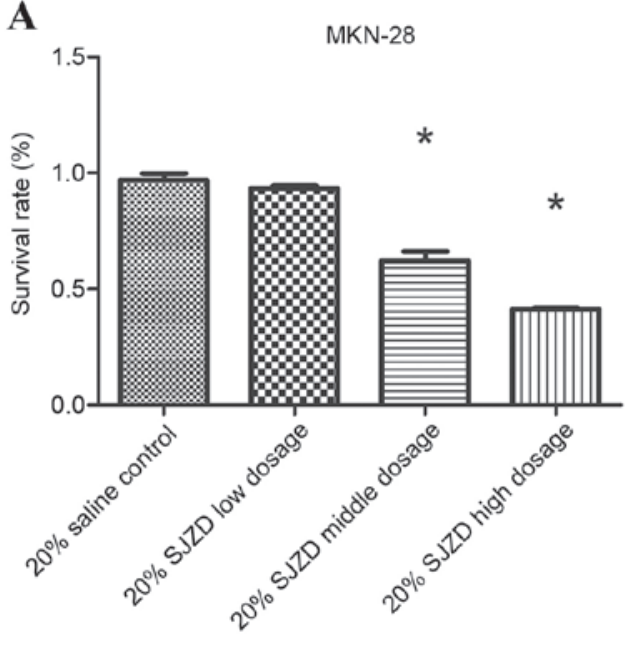

C

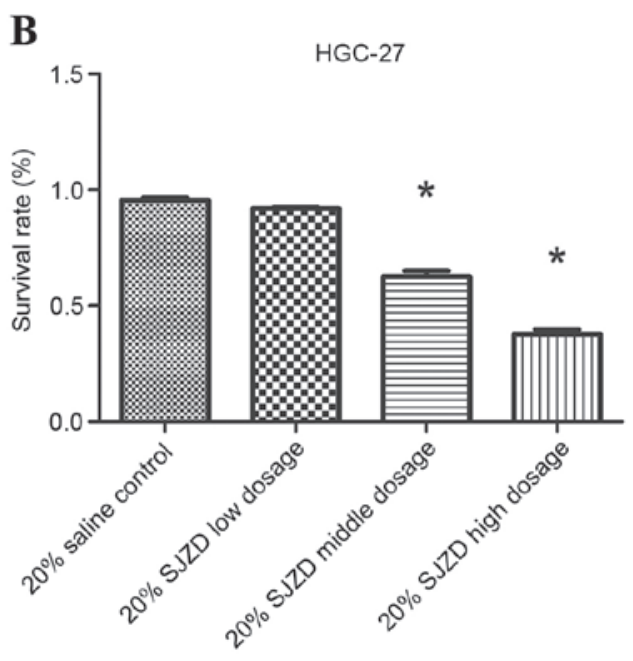

Sijunzi decoction

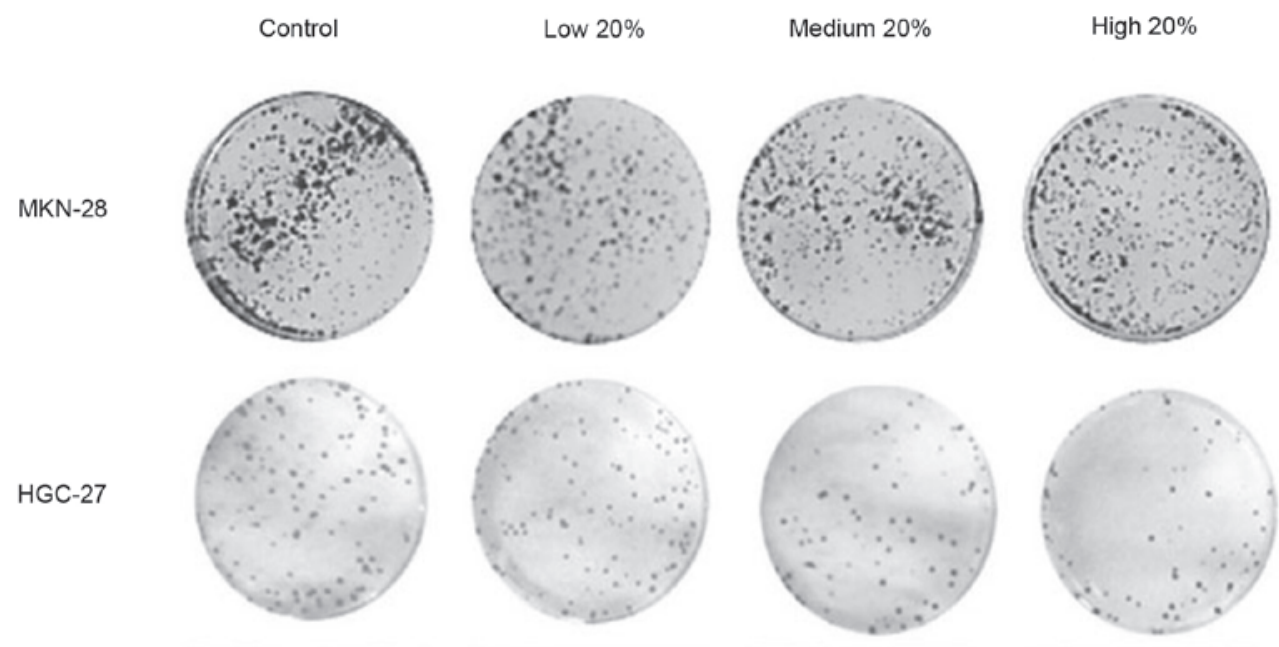

D

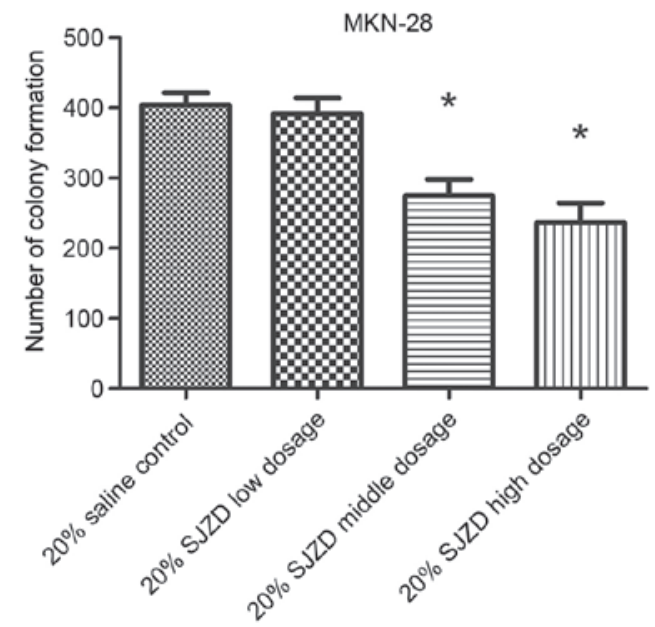

$\mathbf{E}$

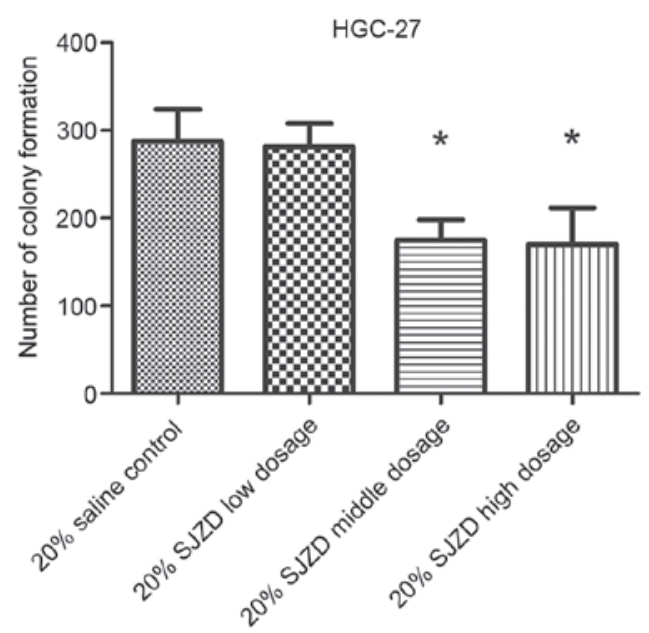

Figure 1. Effect of SJZD-treated rat serum (low, middle and high dosage groups) on the proliferation of (A) MKN-28 and (B) HGC-27 cells. (C) Effect of SJZD-treated rat serum (low, middle and high dosage group) on the colony formation of MKN-28 and HGC-27 cells. Quantification of the (D) MKN-28 and (E) HGC-27 colony formation assay results. * $\mathrm{P}<0.05$ vs. control. SJZD, Sijunzi decoction.

study, they were defined as cells that show higher efflux of the DNA-binding dye Hoechst 33342. The molecules involved in effluxing Hoechst 33342 are members of the
ATP-binding cassette family, such as MDR1 (P-glycoprotein) and ABCG2 (19). For sorting SP cells, nearly confluent cells were harvested by trypsinization with $0.25 \%$ trypsin EDTA 
A
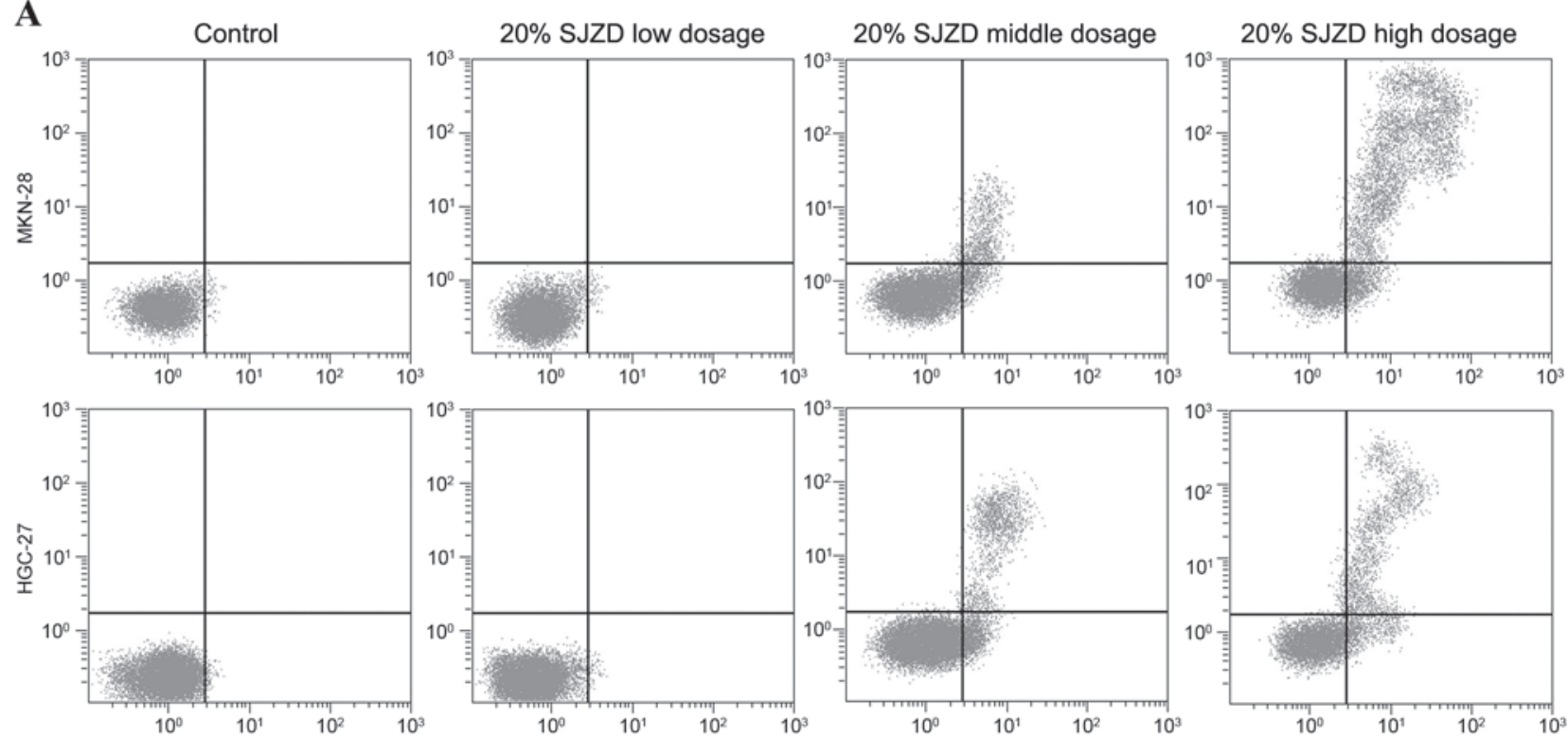

B

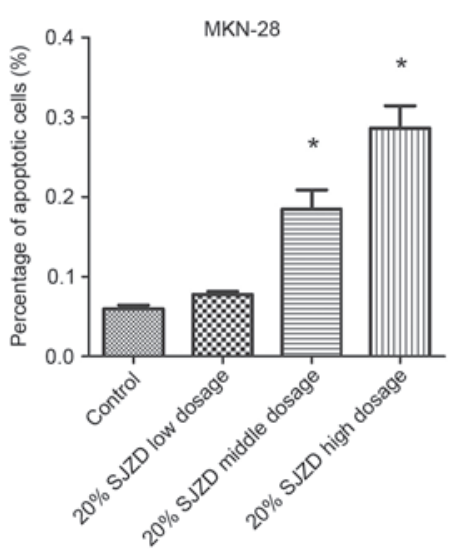

C

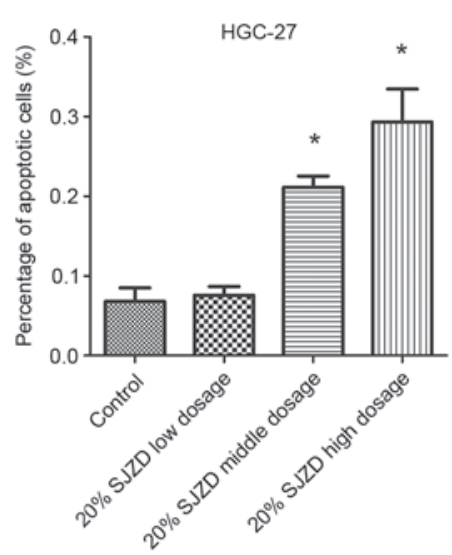

Figure 2. (A) Annexin V/propidium iodide double staining shows cell apoptosis changes in MKN-28 and HGC-27 cells treated with serum from rats treated with different dosages of SJZD. In the cytograms the lower left quadrant includes the viable cells; the lower right quadrant includes early apoptotic cells; the upper right quadrant represents late apoptotic cells; and the upper left quadrant represents necrotic cells. Apoptotic cell death was significantly upregulated ( ${ }^{*} \mathrm{P}<0.05$ vs. the control) in (B) MKN-28 and (C) HGC-27 cells treated with SJZD-treated rat serum (middle and high dosage groups) for 24 h. SJZD, Sijunzi decoction.

(Invitrogen; Thermo Fisher Scientific, Inc.), centrifuged at $500 \mathrm{x} \mathrm{g}$ for $10 \mathrm{~min}$ and washed twice with PBS. They were then re-suspended at a density of $1 \times 10^{6}$ cells $/ \mathrm{ml}$ in pre-warmed $37^{\circ} \mathrm{C}$ RPMI-1640 medium (Gibco; Thermo Fisher Scientific, Inc.) with $2 \% \mathrm{FBS}$ and passed through $40-\mu \mathrm{m}$ cell strainers (BD Falcon; BD Biosciences) to obtain single-cell suspensions. The cells were then divided into two groups. Group 1 was incubated with Hoechst 33342 (Sigma-Aldrich; Merck Millipore) at a final concentration of $5 \mu \mathrm{g} / \mathrm{ml}$ for $70 \mathrm{~min}$ at $37^{\circ} \mathrm{C}$ with gentle agitation every $15 \mathrm{~min}$. Group 2 was pretreated with $100 \mu \mathrm{g} / \mathrm{ml}$ verapamil (Sigma-Aldrich; Merck Millipore) for $15 \mathrm{~min}$ at $37^{\circ} \mathrm{C}$, and then incubated with Hoechst 33342 (final concentration, $5 \mu \mathrm{g} / \mathrm{ml}$ ) for $70 \mathrm{~min}$ at $37^{\circ} \mathrm{C}$ with gentle agitation every $15 \mathrm{~min}$. The incubation process was carried out in the dark. The cells were suspended in ice-cold PBS containing $2 \%$ FBS after staining, and maintained at $4{ }^{\circ} \mathrm{C}$ until flow cytometric analysis. Cells were labeled with $1 \mu \mathrm{g} / \mathrm{ml}$ PI to assess viability $5 \mathrm{~min}$ prior to examination. The stained cells were analyzed using a FACS Aria II system and BD FACS
Diva Software v.6.1.1 (BD Biosciences). The Hoechst dye was excited by an ultraviolet laser at a wavelength of $375 \mathrm{~nm}$, and its fluorescence measured with 450/20 nm (Hoechst blue) and 675/40 LP (long-pass, Hoechst red) optical filters. Regions labeled with Hoechst Red or weak Hoechst blue and negative for verapamil staining was set as the gate for SP cells, and the percentage of SP cells was calculated.

SP cell sorting and identification. SP and non-SP cells were collected separately and re-suspended at 200 cells/well, seeded into 6-well plates and cultured in RPMI-1640 containing $10 \% \mathrm{FBS}$ at $37^{\circ} \mathrm{C}$ with $5 \% \mathrm{CO}_{2}$. After 1 week, $\mathrm{CD} 133$ and ABCG2 expression levels were analyzed by western blotting.

Mitochondrial membrane potential $(\Delta \psi m)$ assay. Loss of $\Delta \psi \mathrm{m}$ is a fundamental early event in the apoptotic process (20). The reagent, 5,5',6,6'-tetrachloro-1,1',3,3'-tetraethylbenzimidazolyl-carbocyanine iodide (JC-1), is the most specific for measuring changes in $\Delta \psi \mathrm{m}$. The high $\Delta \psi \mathrm{m}$ of normal cells 

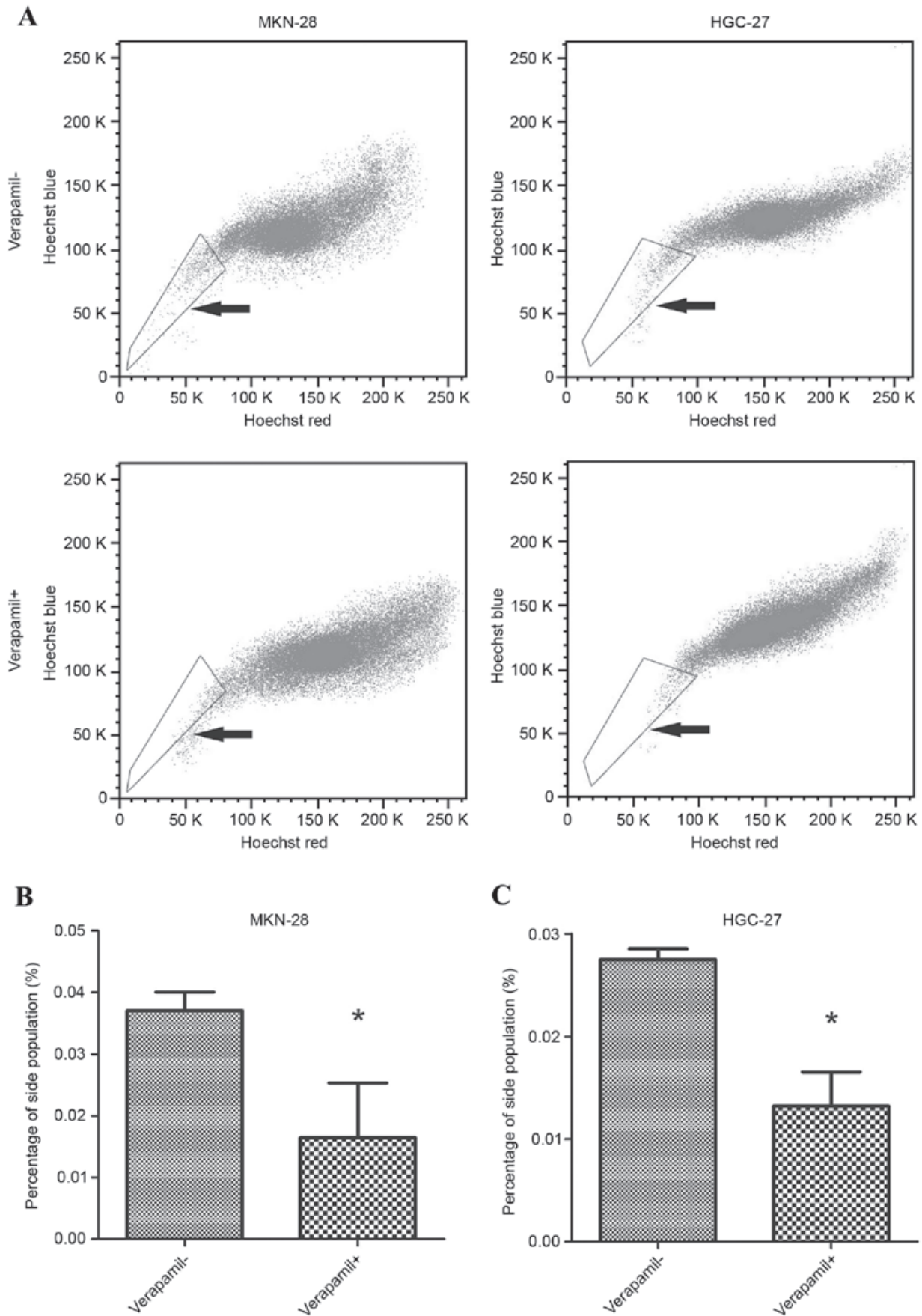

C

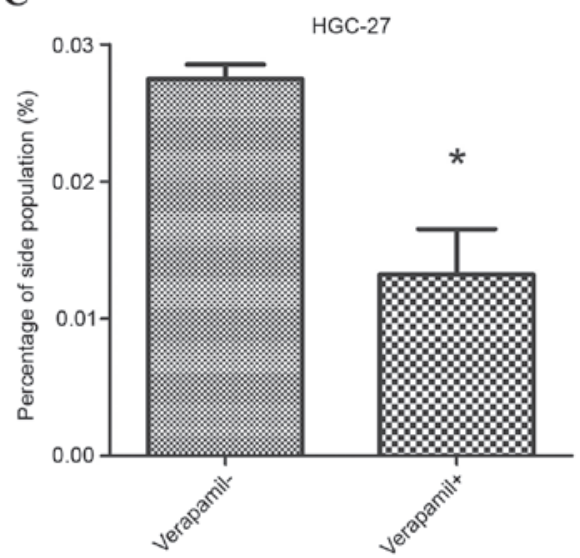

D

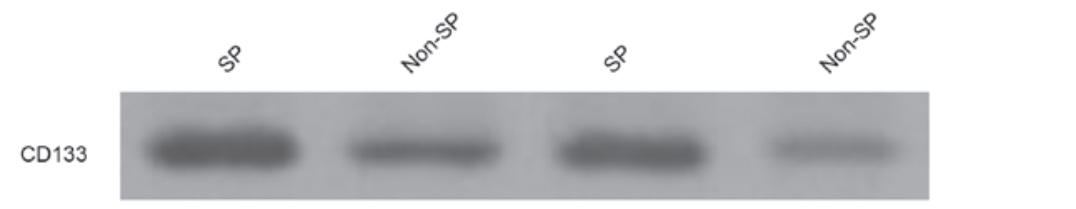

ABCG2

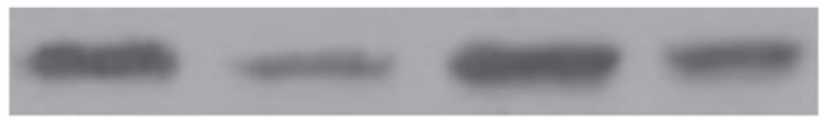

$\beta$-actin

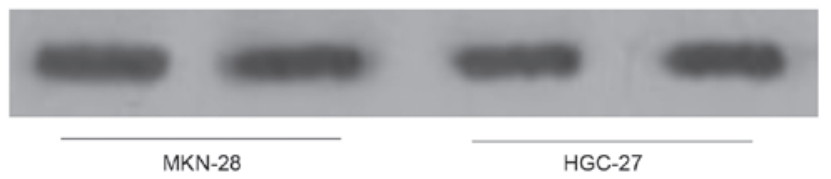

Figure 3. (A) Sorting of SP cells in MKN-28 and HGC-27 cell lines. The outlined area indicated with a black arrow denotes the SP cells. Verapamil treatment significantly reduced the proportion of SP cells in (B) MKN-28 and (C) HGC-27 cells. (D) Western blotting results indicated that the expression levels of CD133 and ABCG2 in SP cells were higher compared with those in non-SP cells. ${ }^{*} \mathrm{P}<0.05$ vs. Verapamil (-). SP, side population. 
A

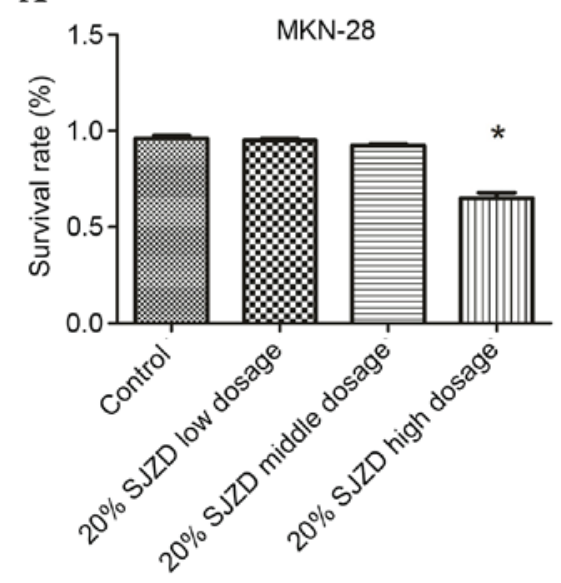

C

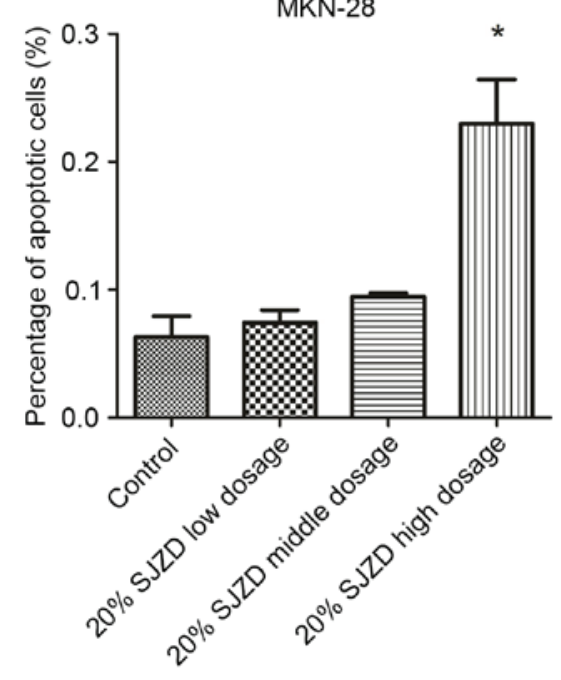

B

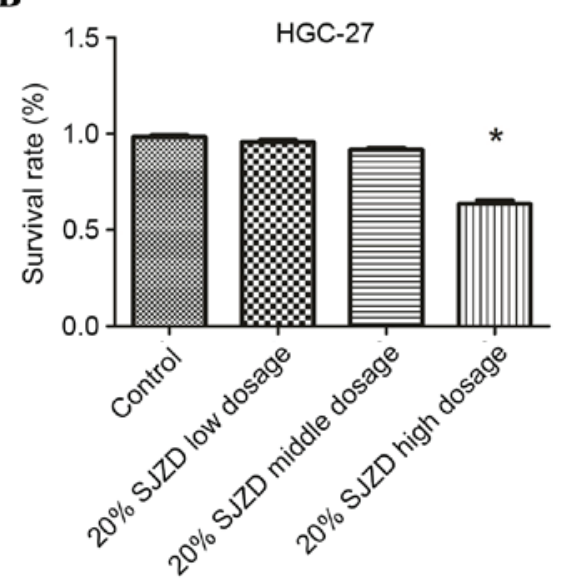

D

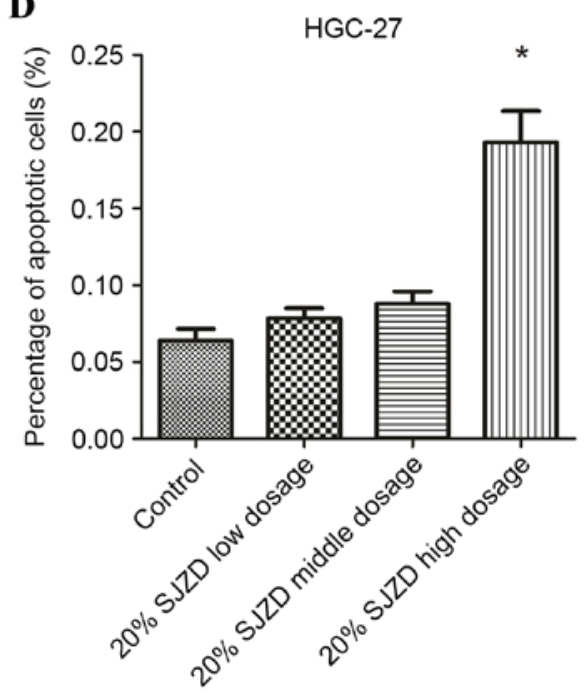

Figure 4. Effect of SJZD-treated rat serum (low, middle and high dosage groups) on the proliferation of SP cells in (A) MKN-28 and (B) HGC-27 cells. Effect of SJZD-treated rat serum (low, middle and high dosage groups) on the apoptosis of SP cells in (C) MKN-28 and (D) HGC-27 cells. * $<<0.05$ vs. control. SJZD, Sijunzi decoction; SP, side population.

loaded with JC-1 allows for the formation of J-aggregates, which exhibits red fluorescence. Upon loss of $\Delta \psi \mathrm{m}$, the J-aggregates dissipate into monomers leading to a shift from red to green fluorescence (21). Therefore, JC-1 was used to assess changes in $\Delta \psi \mathrm{m}$ in the present study. A mitochondrial membrane potential assay kit with JC-1 (Beyotime Institute of Biotechnology) was used. Following treatment with rat serum from each of the four groups (10 or 20\%) for $12 \mathrm{~h}$, $5 \times 10^{5}$ cells were harvested and re-suspended in $0.5 \mathrm{ml}$ media. The suspended cells were incubated with $0.5 \mathrm{ml} \mathrm{JC}-1$ working solution $(1: 160 \mathrm{v} / \mathrm{v})$ at $37^{\circ} \mathrm{C}$ for $20 \mathrm{~min}$. Cells were then rinsed in $1 \mathrm{X} \mathrm{JC}-1$ staining buffer twice, and re-suspended. The fluorescence intensity of JC-1 was measured by flow cytometry with an excitation wavelength of $490 \mathrm{~nm}$ and an emission wavelength of $530 \mathrm{~nm}$ for JC-1 monomers, and excitation wavelength of $525 \mathrm{~nm}$ and an emission wavelength of $590 \mathrm{~nm}$ for JC-1 aggregates.

Western blot analysis. Western blotting was used to analysis protein expression levels. Protein was extracted from the cells using the Mitochondria/Cytosol Fractionation kit (Abcam, Cambridge, UK), according to the manufacturer's instructions, and the concentration was determined by Bradford assay. Equal quantities of protein samples $(30 \mu \mathrm{g})$ were separated by $12 \% \mathrm{SDS}-\mathrm{PAGE}$ and transferred to a $0.2-\mu \mathrm{m}$ polyvinylidene difluoride membrane. The membrane was blocked in $5 \%$ non-fat milk at $25^{\circ} \mathrm{C}$ for $2 \mathrm{~h}$. Membranes were probed with primary antibody $(1 \mu \mathrm{g} / \mathrm{ml})$ overnight at $4^{\circ} \mathrm{C}$. Primary antibodies were as follows: Polyclonal rabbit anti-CD133 IgG (ab19898; Abcam; $1 \mu \mathrm{g} / \mathrm{ml}$ ), monoclonal mouse anti-ABCG2 IgG (sc-18841; Santa Cruz Biotechnology, Inc., Dallas, TX, USA; 1:200), monoclonal mouse anti-Bax IgG (sc-70407; Santa Cruz Biotechnology, Inc.; 1:200), polyclonal rabbit anti-caspase-3 IgG (sc-7148; Santa Cruz Biotechnology, Inc.; 1:200), monoclonal mouse anti-cytochrome C IgG (sc-65396; Santa Cruz Biotechnology, Inc.; 1:200), polyclonal rabbit anti-PARP IgG (sc-7150; Santa Cruz Biotechnology, Inc.; 1:200), monoclonal mouse anti-COX IV IgG (sc-376731; Santa Cruz Biotechnology, Inc.; 1:100), monoclonal mouse anti- $\beta$-actin IgG (sc-47778; Santa Cruz Biotechnology, Inc.; 1:200). Membranes were then washed three times in TBST and incubated with goat anti-mouse horseradish peroxidase (HRP) conjugated secondary antibody (sc-2005; Santa Cruz Biotechnology, Inc.; 1:2,000) or goat anti-rabbit 
$\mathbf{A}$

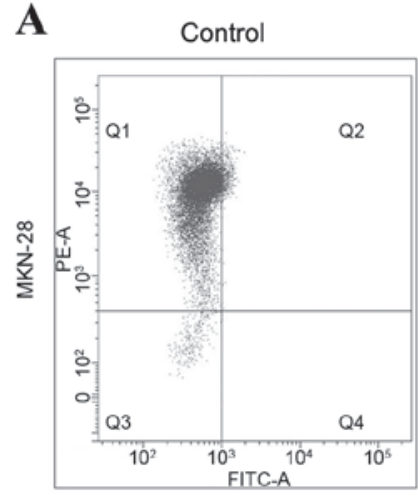

$20 \%$ SJZD low dosage
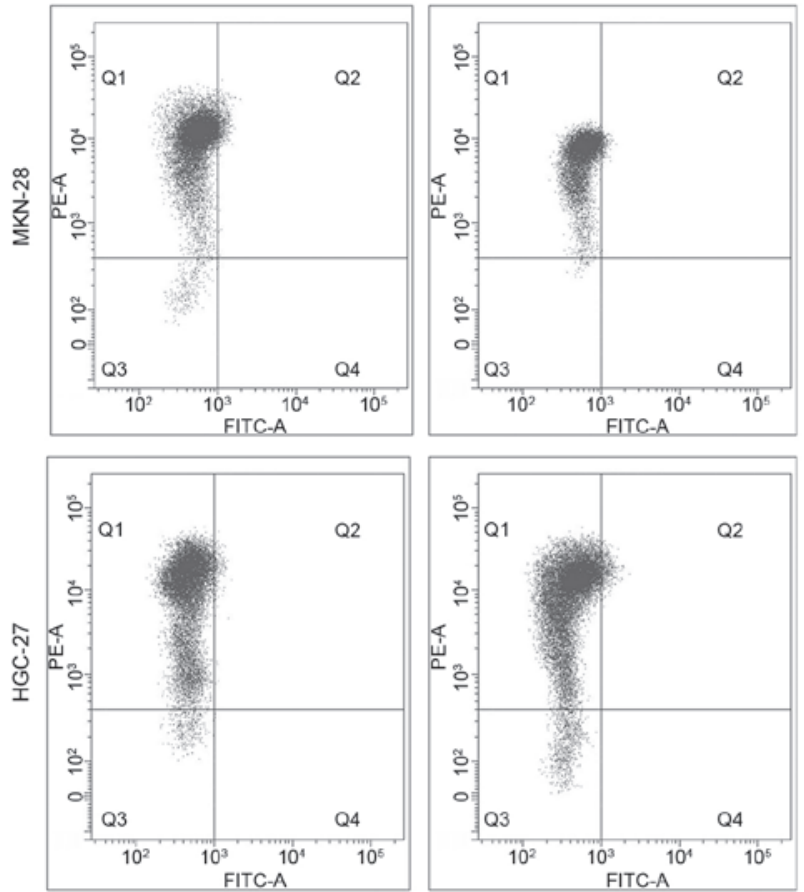

B

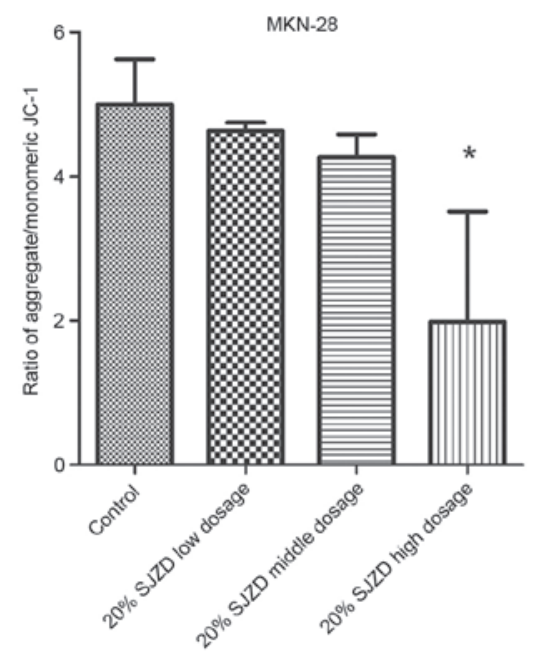

D

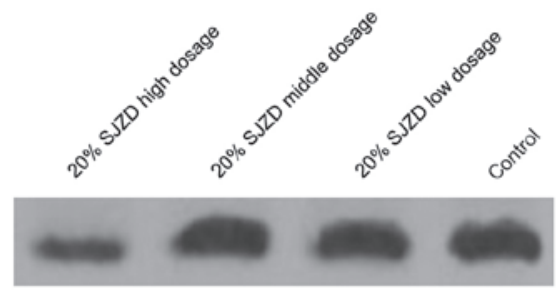

COXIV

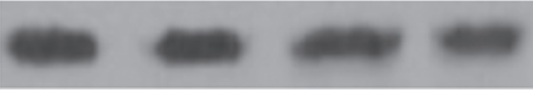

Cytosolic cyto C

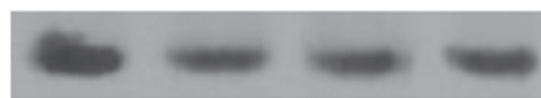

$\beta$-actin

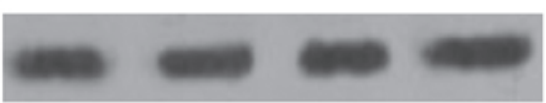

MKN-28
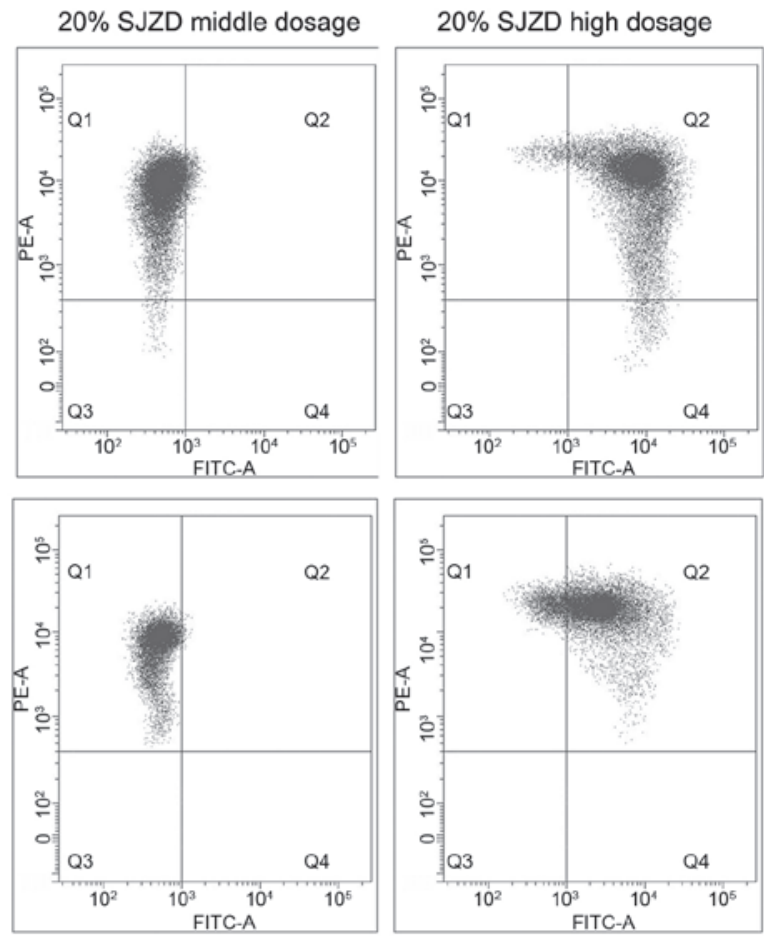

C
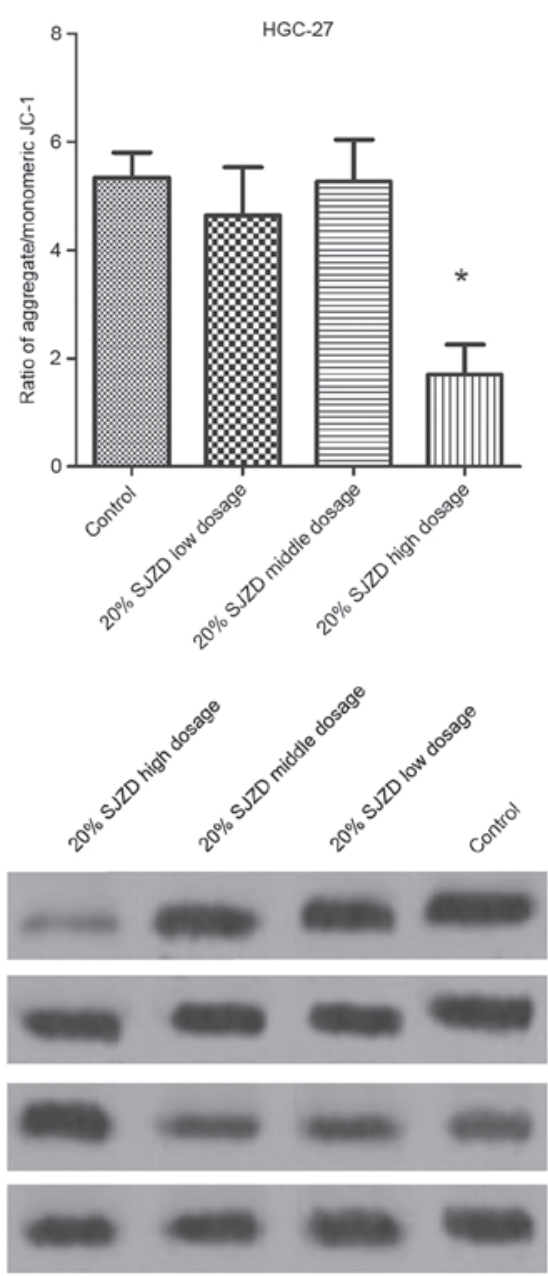

HGC-27

Figure 5. (A) Mitochondrial membrane potential in MKN-27 and HGC-27 cells exposed to the serum of rats treated with different doses of SJZD as determined by flow cytometry. The $20 \%$ high dosage SJZD-treated rat serum decreased mitochondrial membrane potential in (B) MKN-28 and (C) HGC-27 cells. (D) Western blotting results showed that 20\% high dosage SJZD-treated rat serum increased cytosolic cytochrome $c$ content and decreased mitochondrial cytochrome $c$ content. ${ }^{*} \mathrm{P}<0.05$ vs. control. SJZD, Sijunzi decoction. 


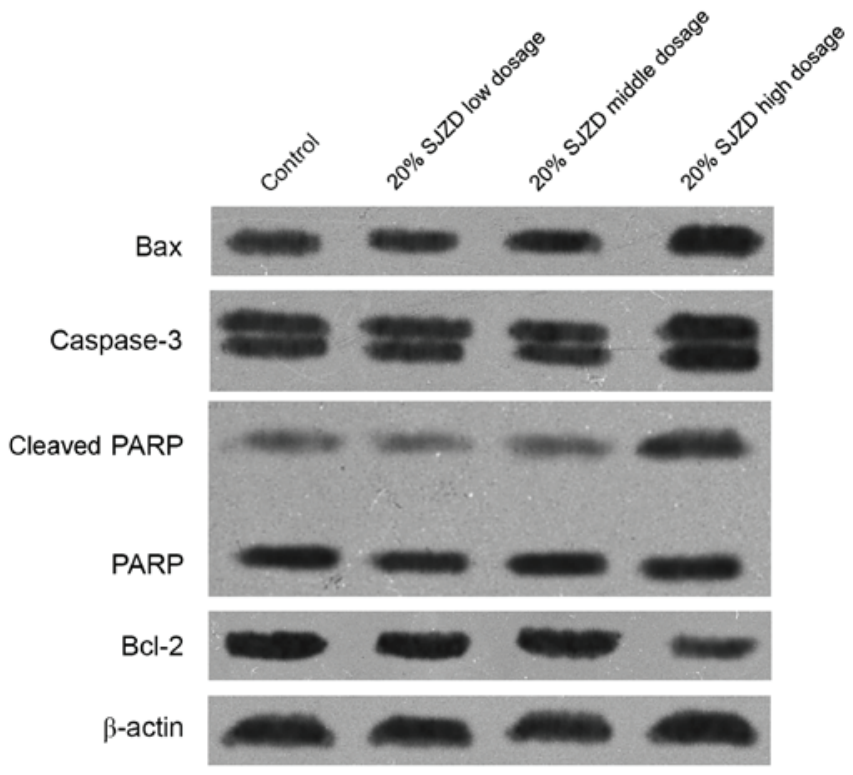

Figure 6. Western blotting results in MKN-28 side population cells exposed to the serum of rats treated with different doses of SJZD. The expression levels of Bax, cleaved caspase-3 and cleaved PARP protein increased significantly, while that bcl-2 decreased following treatment with the serum. SJZD, Sijunzi decoction.

HRP-conjugated secondary antibody (sc-2004; Santa Cruz Biotechnology, Inc.; 1:5,000) for $1 \mathrm{~h}$ at room temperature and then washed three times in TBST. The signals were visualized using an enhanced chemiluminescence solution (ECL Plus; Amersham Pharmacia Biotech; GE Healthcare Life, Chalfont, UK) and were exposed to X-Omat LS film (Eastman Kodak Co., Rochester, NY, USA). CIX IV and $\beta$-actin were used as cytoplasmic and mitochondrial markers, respectively.

Statistical analysis. All statistical analyses were performed using SPSS 17.0 software (SPSS, Inc., Chicago, IL, USA) and data expressed as mean \pm standard deviation. For comparison of the means of two groups, Student's t-test was used. For comparison of the magnitude of changes among three or more groups, one-way analysis of variance was used with Student-Newman-Keuls and Least Significant Difference post hoc tests. All experiments reported in this study were performed independently at least three times. $\mathrm{P}<0.05$ was considered to indicate a statistically significant difference.

\section{Results}

SJZD-treated rat serum significantly inhibits the proliferation and colony formation of MKN-28 and HGC-27 cells. In order to mimic the pharmacodynamics of SJZD in vivo, SJZD-treated rat serum was used in the present study. Firstly, the inhibitory effect of SJZD-treated rat serum on the proliferation of gastric carcinoma cell lines was investigated. The results of the MTT assay showed that serum from the middle and high dosage groups (20\%) significantly inhibited the proliferation of MKN-28 cells (LSD test: $\mathrm{P}=0.002$ for $20 \%$ middle dose, $\mathrm{P}<0.001$ for $20 \%$ high dose) and HGC-27cells (LSD test: $\mathrm{P}=0.003$ for $20 \%$ middle dose, $\mathrm{P}<0.001$ for $20 \%$ high dose) (Fig. 1A and B), while there was no significant difference in growth rate between the low dosage group and the control. In addition, following treatment with $20 \%$ SJZD serum from the middle and high dosage groups, MKN-28 cells (LSD test: $\mathrm{P}=0.007$ for $20 \%$ middle dose, $\mathrm{P}<0.001$ for $20 \%$ high dose) and HGC-27 cells (LSD test: $\mathrm{P}<0.001$ for $20 \%$ middle dose, $\mathrm{P}<0.001$ for $20 \%$ high dose) also exhibited a significantly decreased capability for colony formation compared with the control group (Fig. 1C-E). The 10\% SJZD serum had no significant inhibitory effects on proliferation or colony formation in MKN-28 and HGC-27 cells (data not shown).

SJZD-treated rat serum induces apoptosis in MKN-28 and HGC-27 cells. The apoptotic effects of SJZD-treated rat serum on gastric carcinoma cell lines were investigated. Flow cytometric analysis of cells treated with serum containing SJZD showed that only serum from the $20 \%$ middle and high dosage groups induced apoptosis in $\mathrm{MKN}-28$ cells $(\mathrm{P}=0.001$ for $20 \%$ middle dose, $\mathrm{P}<0.001$ for $20 \%$ high dose) and HGC-27 cells $(\mathrm{P}<0.001$ for $20 \%$ middle dose, $\mathrm{P}<0.001$ for $20 \%$ high dose) (Fig. 2). Apoptosis was not observed in cells treated with SJZD at the low dosage. The $10 \%$ SJZD serum had no significant effects on apoptosis in MKN-28 and HGC-27 cells (data not shown).

SP cells exist in MKN-28 and HGC-27 cell lines. Hoechst 33342 staining demonstrated that SP cells exist in the MKN-28 and HGC-27 cell lines, with percentages of $3.71 \pm 0.30$ and $1.65 \pm 0.88 \%$, respectively. When subjected to verapamil treatment, the proportion of SP cells in the two cell lines decreased significantly, to $2.75 \pm 0.10(\mathrm{P}=0.019)$ and $1.32 \pm 0.33 \%(\mathrm{P}=0.002)$, respectively (Fig. 3A-C). The SP cells in the MKN-28 and HGC-27 cell lines were then separated. Western blot analysis of the separated SP cells revealed the evident enrichment of SP cell markers CD133 and ABCG2 compared with their levels in non-SP cells (Fig. 3D).

SJZD-treated rat serum induces apoptosis in SP cells of $M K N-28$ and $H G C-27$ cell lines. The antiproliferative effect of SJZD in the SP cells was investigated. As shown by MTT assay and apoptosis analysis, only serum from the $20 \%$ high dosage group significantly inhibited MKN-28 (P=0.002) and HGC-27 SP cell $(\mathrm{P}<0.001)$ proliferation and induced apoptosis in these cells, while apoptosis was not observed in the other groups (Fig. 4). The 10\% SJZD serum had no significant effects on apoptosis in SP cells of MKN-28 and HGC-27 cell lines (data not shown).

SJZD-treated rat serum induces $\Delta \psi m$ collapse in $M K N-28$ and HGC-27 SP cells. As shown by $\Delta \psi m$ assay (Fig. 5A-C), the mitochondria in SP cells treated with $20 \%$ SJZD-treated rat serum from the low and middle dosage groups maintained a high membrane potential with strong red fluorescence. However, the addition of $20 \%$ high dosage SJZD-treated rat serum caused a reduction of the red:green fluorescence ratio, which reflected the collapse of $\Delta \psi m(\mathrm{P}<0.05)$ in SJZD-treated cells. Western blotting results indicated that following treatment with $20 \%$ high dosage SJZD-treated rat serum for $24 \mathrm{~h}$, cytochrome $c$ content in the mitochondria was significantly reduced, while the content in the cytoplasm was significantly increased, indicating that high dosage SJZD-treated rat serum 
promoting the release of cytochrome $c$ from mitochondria to cytosol (Fig. 5D).

SJZD-treated rat serum induces MKN-28 and HGC-27 SP cell apoptosis via the endogenous apoptosis pathway. To further investigate the mechanism by which SJZD promotes apoptosis in MKN-28 SP cells, the expression of apoptosis-related proteins Bax, Bcl-2, caspase-3 and PARP in SP cells was evaluated in the cytosol fraction (Fig. 6). The results showed that after $48 \mathrm{~h}$ treatment with $20 \%$ high dose SJZD serum, Bax was markedly upregulated and Bcl-2 was downregulated. Furthermore, the PARP level was clearly elevated while the level of Bcl-2 was notably decreased. Moreover, the expression of active fragments of caspase- 3 and PARP, the substrate of caspase-3, was also detected in the cytosol.

\section{Discussion}

Gastric cancer is one of the leading causes of mortality worldwide. Although the incidence rate is gradually decreasing, the overall survival rate of patients with gastric cancer remains low $(22,23)$. In China, it is the second most common malignant tumor type and its incidence continues to rise (24). For patients with gastric cancer who are unable to receive surgical treatment or are affected by recurrence and metastasis, chemotherapy is the only therapeutic option. However, the serious side effects of chemotherapeutic drugs drives the search to identify new methods for the treatment of gastric cancer (25). CMs have been used for the prevention and treatment of human cancers in Asian societies for centuries, and are considered as important strategies for the treatment of cancer (26-28). SJZD is a well-known herbal formulation that is considered mild in nature. It has been reported that the formulation has physiological functions, such as improving gastrointestinal function, strengthening the immune system, and speeding up the production of red blood cells (29). This formulation can also be used for the treatment of malignant tumors (9). However, in the context of evidence-based medicine, the determination of efficacy requires critical evaluation using modern medical research approaches. Due to its complex composition, the anticancer mechanism of Sijunzi decoction has not yet been clarified. This is the reason why many effective CMs are not able to attain international recognition. In the current study, the serum pharmacology method was used to extract medicated serum from animals treated with three different concentrations of SJZD.

MKN-28 and HGC-27 cells were used as in vitro models to explore the anticancer mechanism of SJZD. It was found that serum from SJZD-treated rats significantly inhibited the growth and colony formation capacity of MKN-28 and HGC-27 cells. Flow cytometric cell sorting indicated that $\mathrm{SP}$ cells were present in the MKN-28 and HGC-27 cell lines. Following the suggestion of the concept of cancer stem cells, treatments against cancer have begun to target cancer stem cells since certain oncologists consider cancer stem cells to be the cause of resistance and relapse (30). The SP has been identified as a subpopulation of cancer cells with high proliferative and drug-resistant capacities (31). Several cancer stem cell markers, such as CD44 and CD24 for breast cancer stem cells have been subsequently discovered in SP cells (32). The high expression level of the drug transport protein ABCG2 in SP cells enables them to quickly pump out Hoechst fluorescent dye (19). SP cells have been found in a variety of tumor tissues, such as lung cancer, pancreatic cancer, liver cancer and colon cancer tissues (33-36). It has been reported that SP cells also exist in gastric cancer tissues, and CD133 and ABCG2 are markers for gastric cancer SP cells $(37,38)$. In the present study, a proportion of SP cells were found in the MKN-28 and HGC-27 cell lines, and CD133 and ABCG2 protein expression levels were significantly higher in the SP cells than in non-SP cells, which further confirmed that they were SP cells.

As indicated by the MTT and apoptosis results in the present study, SJZD-treated rat serum significantly inhibited the growth of SP cells and induces the apoptosis of SP cells. Cell apoptosis may be physiological or pathological. The apoptosis process involves the activation, expression and regulation of a series of genes; many signaling pathways are also involved, such as the mitochondrial apoptosis pathway, death receptor apoptosis pathway and endoplasmic reticulum apoptosis pathway $(39,40)$. In the present study, it was found that SJZD-containing serum can induce $\Delta \psi m$ collapse, reduce mitochondrial cytochrome $c$, increase the $\mathrm{Bax} / \mathrm{Bcl}-2$ protein expression ratio and cytoplasmic cytochrome $c$ content, and activate caspase-3 and PARP, which suggests that the apoptosis of SP cells induced by SJZD occurred through activation of the mitochondrial apoptotic pathway.

In summary, the present study found that SP cells exist in the MKN-28 and HGC-27 cell lines, while SJZD-treated rat serum is able to induce apoptosis and inhibit the proliferation of SP cells through activation of the mitochondrial apoptotic pathway. This may be the underlying antitumor mechanism of SJZD. However, even though the serum is effective, it would be advantageous to identify the effective molecular species in SJZD using chromatographic techniques to provide greater effects and better quality control, in order to obtain the maximum effectiveness of SJZD.

\section{Acknowledgements}

The present study was supported by Key Program of Bengbu Medical College (grant no. Bykf12A05), Key Program of Foundation for Youth Talents in Colleges and Universities of Anhui Province (grant no. 2013SQRL052ZD) and Key Program of Natural Science Foundation in Colleges and Universities of Anhui Province (grant no. KJ2017A240).

\section{References}

1. Qi F, Li A, Inagaki Y, Gao J, Li J, Kokudo N, Li XK and Tang W: Chinese herbal medicines as adjuvant treatment during chemoor radio-therapy for cancer. Biosci Trends 4: 297-307, 2010.

2. Xu W, Towers AD, Li P and Collet JP: Traditional Chinese medicine in cancer care: Perspectives and experiences of patients and professionals in China. Eur J Cancer Care (Engl) 15: 397-403, 2006.

3. Chen GQ, Shi XG, Tang W, Xiong SM, Zhu J, Cai X, Han ZG, Ni JH, Shi GY, Jia PM, et al: Use of arsenic trioxide (As2O3) in the treatment of acute promyelocytic leukemia (APL): I. As2O3 exerts dose-dependent dual effects on APL cells. Blood 89: 3345-3353, 1997

4. Nam S, Scuto A, Yang F, Chen W, Park S, Yoo HS, Konig H, Bhatia R, Cheng X, Merz KH, et al: Indirubin derivatives induce apoptosis of chronic myelogenous leukemia cells involving inhibition of Stat5 signaling. Mol Oncol 6: 276-283, 2012. 
5. Xiao Z, Hao Y, Liu B and Qian L: Indirubin and meisoindigo in the treatment of chronic myelogenous leukemia in China. Leuk Lymphoma 43: 1763-1768. 2002

6. Liu T, Song Y, Chen H, Pan S and Sun X: Matrine inhibits proliferation and induces apoptosis of pancreatic cancer cells in vitro and in vivo. Biol Pharm Bull 33: 1740-1745, 2010.

7. Wang HQ, Jin JJ and Wang J: Matrine induces mitochondrial apoptosis in cisplatin-resistant non-small cell lung cancer cells via suppression of $\beta$-catenin/survivin signaling. Oncol Rep 33: 2561-2566, 2015.

8. Yu P, Liu Q, Liu K, Yagasaki K, Wu E and Zhang G: Matrine suppresses breast cancer cell proliferation and invasion via VEGF-Akt-NF-kappaB signaling. Cytotechnology 59: 219-229, 2009.

9. Yang N, Han F, Cui H, Huang J, Wang T, Zhou Y and Zhou J: Matrine suppresses proliferation and induces apoptosis in human cholangiocarcinoma cells through suppression of JAK2/STAT3 signaling. Pharmacol Rep 67: 388-393, 2015.

10. Lin H, Liu J and Zhang Y: Developments in cancer prevention and treatment using traditional Chinese medicine. Front Med 5 : 127-133, 2011

11. Zhong WR, Huang YX and Cui JP: Clinical study on modified sijunzi decoction in treating intestinal metaplasia of gastric mucosa. Zhongguo Zhong Xi Yi Jie He Za Zhi 17: 462-464, 1997 (In Chinese).

12. Wu B and Xuan ZR: Progress in research on applying Sijunzi Decoction in treating digestive malignant tumor. Chin J Integr Med 13: 156-159, 2007.

13. Zhao AG, Zhao HL, Jin XJ, Yang JK and Tang LD: Effects of Chinese Jianpi herbs on cell apoptosis and related gene expression in human gastric cancer grafted onto nude mice. World J Gastroenterol 8: 792-796, 2002.

14. Bochu W, Liancai Z and Qi C: Primary study on the application of Serum Pharmacology in Chinese traditional medicine. Colloids Surf B Biointerfaces 43: 194-197, 2005.

15. Wu DH, Xu L, Wen CP, Xie GQ, Ji JJ, Pan JL, Jiao YF and Fan YS: The effects of Jieduquyuzishen prescription-treated rat serum on the BAFF/BAFF-R signal pathway. PLoS One 10 e0118462, 2015

16. Cao Y, Liu F, Huang Z and Zhang Y: Protective effects of Guanxin Shutong capsule drug-containing serum on tumor necrosis factor- $\alpha$-induced endothelial dysfunction through nicotinamide adenine dinucleotide phosphate oxidase and the nitric oxide pathway. Exp Ther Med 8: 998-1004, 2014.

17. Jiang YR, Miao Y, Yang L, Xue M, Guo CY, MaXJ, Yin HJ, Shi DZ and Chen KJ: Effect of chinese herbal drug-containing serum for activating-blood and dispelling-toxin on ox-LDL-induced inflammatory factors' expression in endothelial cells. Chin J Integr Med 18: 30-33, 2012

18. Hu C, Li H, Li J, Zhu Z, Yin S, Hao X, Yao M, Zheng S and $\mathrm{Gu} \mathrm{J}$ : Analysis of ABCG2 expression and side population identifies intrinsic drug efflux in the HCC cell line MHCC-97L and its modulation by Akt signaling. Carcinogenesis 29: 2289-2297, 2008 .

19. Scharenberg CW, Harkey MA and Torok-Storb B: The ABCG2 transporter is an efficient Hoechst 33342 efflux pump and is preferentially expressed by immature human hematopoietic progenitors. Blood 99: 507-512, 2002.

20. Ly JD, Grubb DR and Lawen A: The mitochondrial membrane potential (deltapsi(m)) in apoptosis; an update. Apoptosis 8 115-128, 2003.

21. Zuliani T, Duval R, Jayat C, Schnébert S, André P, Dumas M and Ratinaud MH: Sensitive and reliable JC-1 and TOTO-3 double staining to assess mitochondrial transmembrane potential and plasma membrane integrity: Interest for cell death investigations. Cytometry A 54: 100-108, 2003.
22. Jemal A, Bray F, Center MM, Ferlay J, Ward E and Forman D: Global cancer statistics. CA Cancer J Clin 61: 69-90, 2011.

23. Siegel R, Ma J, Zou Z and Jemal A: Cancer statistics, 2014 CA Cancer J Clin 64: 9-29, 2014.

24. Chen W, Zheng R, Baade PD, Zhang S, Zeng H, Bray F, Jemal A, $\mathrm{Yu}$ XQ and He J: Cancer statistics in China, 2015. CA Cancer J Clin 66: 115-132, 2016.

25. Dikken JL, van de Velde CJ, Coit DG, Shah MA, Verheij M and Cats A: Treatment of resectable gastric cancer. Therap Adv Gastroenterol 5: 49-69, 2012.

26. Yang G, Li X, Li X, Wang L, Li J, Song X, Chen J, Guo Y, Sun X, Wang S, et al: Traditional chinese medicine in cancer care: A review of case series published in the chinese literature. Evid Based Complement Alternat Med 2012: 751046, 2012.

27. Ma X, Lin $C$ and Zhen W: Cancer care in China: A general review. Biomed Imaging Interv J 4: e39, 2008.

28. Liu J, Li X, Liu J, Ma L, Li X and Fønneb $\varnothing$ V: Traditional Chinese medicine in cancer care: A review of case reports published in Chinese literature. Forsch Komplementmed 18: 257-263, 2011.

29. Lu Y, Lin H, Zhang J, Wei J, Sun J and Han L: Sijunzi Decoction attenuates 2, 4, 6-trinitrobenzene sulfonic acid (TNBS)-induced colitis in rats and ameliorates TNBS-induced claudin-2 damage via $\mathrm{NF}-\kappa \mathrm{B}$ pathway in $\mathrm{Caco} 2$ cells. BMC Complement Altern Med 17: 35, 2017.

30. Sharma B and Singh RK: Emerging candidates in breast cancer stem cell maintenance, therapy resistance and relapse. J Carcinog 10: 36, 2011.

31. Eaves CJ: Cancer stem cells: Here, there, everywhere? Nature 456: 581-582, 2008.

32. Tanaka H, Nakamura M, Kameda C, Kubo M, Sato N, Kuroki S, Tanaka $\mathrm{M}$ and Katano M: The Hedgehog signaling pathway plays an essential role in maintaining the CD44+CD24-/low subpopulation and the side population of breast cancer cells. Anticancer Res 29: 2147-2157, 2009.

33. Ho MM, Ng AV, Lam S and Hung JY: Side population in human lung cancer cell lines and tumors is enriched with stem-like cancer cells. Cancer Res 67: 4827-4833, 2007.

34. Chiba T, Kita K, Zheng YW, Yokosuka O, Saisho H, Iwama A, Nakauchi $\mathrm{H}$ and Taniguchi H: Side population purified from hepatocellular carcinoma cells harbors cancer stem cell-like properties. Hepatology 44: 240-251, 2006.

35. Zhang SN, Huang FT, Huang YJ, Zhong W and Yu Z: Characterization of a cancer stem cell-like side population derived from human pancreatic adenocarcinoma cells. Tumori 96: 985-992, 2010.

36. Sussman RT, Ricci MS, Hart LS, Sun SY and El-Deiry WS: Chemotherapy-resistant side-population of colon cancer cells has a higher sensitivity to TRAIL than the non-SP, a higher expression of c-Myc and TRAIL-receptor DR4. Cancer Biol Ther 6: 1490-1495, 2007

37. Schmuck R, Warneke V, Behrens HM, Simon E, Weichert W and Röcken C: Genotypic and phenotypic characterization of side population of gastric cancer cell lines. Am J Pathol 178: 1792-1804, 2011.

38. Fukuda K, Saikawa Y, Ohashi M, Kumagai K, Kitajima M, Okano H, Matsuzaki Y and Kitagawa Y: Tumor initiating potential of side population cells in human gastric cancer. Int J Oncol 34: 1201-1207, 2009.

39. Breckenridge DG, Germain M, Mathai JP, Nguyen $M$ and Shore GC: Regulation of apoptosis by endoplasmic reticulum pathways. Oncogene 22: 8608-8618, 2003.

40. Ferri KF and Kroemer G: Organelle-specific initiation of cell death pathways. Nat Cell Biol 3: E255-E263, 2001. 